\title{
Nonlinear Estimation of Synaptic Conductances via Piecewise Linear Systems
}

\author{
Antoni Guillamon, Rafel Prohens, Antonio E. Teruel, and Catalina Vich
}

\begin{abstract}
We use the piecewise linear McKean model to present a proof-of-concept to address the estimation of synaptic conductances when a neuron is spiking. Using standard techniques of non-smooth dynamical systems, we obtain an approximation of the period in terms of the parameters of the system which allows to estimate the steady synaptic conductance of the spiking neuron. The method gives also fairly good estimations when the synaptic conductances vary slowly in time.
\end{abstract}

\section{Introduction}

The problem addressed in this manuscript is framed into the challenge of unveiling the functional connectivity in the brain; that is, to obtain information about strength and timing of the input currents (mediated by synapses) that a single neuron (the post-synaptic cell) receives from others (the pre-synaptic ones). In particular, we aim at disentangling the arrangement of excitation versus inhibition impinging on the cell. We will focus on the estimation of the conductances of these currents, a quantity which is not directly measurable but needs to be extracted from experimentally

\footnotetext{
A. Guillamon ( $\square)$

Departament de Matemàtiques-EPSEB, Universitat Politècnica de Catalunya,

Barcelona, Spain

e-mail: antoni.guillamon@upc.edu

R. Prohens · A.E. Teruel · C. Vich

Departament de Matemàtiques i Informàtica, Universitat de les Illes Balears,

Palma, Spain

e-mail: rafel.prohens@uib.cat
}
A.E. Teruel
e-mail: antonioe.teruel@uib.es
C. Vich
e-mail: catalina.vich@uib.es 
accessible data like the membrane potential of the neuron. For a review of the relevant concepts in neuroscience, we refer the reader to the book Ermentrout-Terman [4], and the introduction in Vich-Guillamon [8] for a quick survey on the problem of estimation of conductances. A major hindrance in this problem is the estimation when ionic currents are active, specially in spiking regimes, where ionic conductances are much higher than synaptic ones; see Guillamon-McLaughlin-Rinzel [6]. No methods are yet known to estimate synaptic conductances in this paradigm. From a mathematical point of view, this is indeed an inverse problem consisting of estimating some parameters from time courses of one variable of the associated dynamical system. In this work, we aim at giving a theoretical proof-of-concept by considering a simplified model of neuronal activity, namely a non-smooth caricature of the FitzHugh-Nagumo model (see Coombes-Thul-Wedgwood [3]), which allows a very sharp approximation of the nonlinear $f-I$ curve thanks to special properties of piecewise linear systems. Moreover, the same idea could be applied to smooth systems with approximate expressions of the corresponding $f-I$ curves. The model we consider is a version of the McKean model as described in Coombes [2], where the synaptic current, $I_{s y n}(v)$, has been considered apart from the rest of external currents, $I$. That is, we consider the system

$$
\left\{\begin{array}{l}
C \dot{v}=f(v)-w-w_{0}+I-I_{s y n}(v), \\
\dot{w}=v-\gamma w-v_{0}
\end{array}\right.
$$

where $I_{s y n}(v)=g_{s y n}\left(v-v_{\text {syn }}\right)$ and $f(v)$ is defined in a continuous but non-differentiable way according to three zones of the phase space, left, middle and right: $f(v)=f_{L}(v):=-v$ if $v<a / 2, f(v)=f_{M}(v):=v-a$ if $a / 2 \leq v \leq$ $(1+a) / 2$, and $f(v)=f_{R}(v):=1-v$ if $v>(1+a) / 2$. For the significance of the parameters involved in the model, see Coombes [2]. Here, we focus on the most relevant parameters for this work. We consider to have a single source of synaptic conductances, which is modeled by means of $v_{s y n}$, the synaptic reversal potential, and $g_{\text {syn }}>0$, the synaptic conductance. The ionic currents are modeled through the expression $f(v)-w-w_{0}$ in the first equation of (1), designed to mimic the qualitative behaviour of the $\mathrm{N}$-shaped $v$-nullcline of more biophysically realistic models. Since $C$, the parameter related to cell membrane capacitance, is assumed to be small and bounded, $0<C \ll 0.1$, system (1) is then a slow-fast dynamical system, where $v$ is the fast variable and $w$ is the slow one.

In Abbott [1] and Tonnelier-Gerstner [7], the authors show the existence of two values $I_{1}<I_{2}$ for the applied current for which system (1) with $I_{s y n}(v)=0$ exhibits a periodic orbit, which is unique, if and only if $I_{1}<I<I_{2}$. In Coombes [2], approximations of the period $T$ were obtained, mainly based on the slow-fast nature of the system. More recently, in Fernández-García-Desroches-Krupa-Clément [5], the authors provide an approximate expression for the period $T$ by taking advantage of the slow invariant manifolds for $C \ll 1$. We show that the previous scenario persists for $I_{s y n}(v) \neq 0$ and that the interval where periodic orbits exist depends also on $g_{s y n}$ as well as the period, $T=T\left(C, I, g_{s y n}\right)$. We present a new approximation of 
$T, \hat{T}$, which includes this dependence on $g_{s y n}$ and, moreover, improves the existing approximations. The key points are two refinements: the consideration of the flight time in the central region, and a special projection on the slow manifold.

In the range of applied currents in which the neuron is spiking, we have found numerical evidence that the period of this oscillation has a nonlinear but monotonic dependence on $g_{s y n}$. Hence, as a consequence of this monotonicity, by knowing $\hat{T}$ and the applied current $I$ (i.e., knowing the so-called $f-I$ curve), we can compute $g_{s y n}$ by solving numerically a non-linear equation with a unique solution, and thus we are able to estimate the steady synaptic conductance of the neuron, which is the goal of this work.

\section{Main Result}

It is easy to check that the system (1) with $f(v)=f_{L}(v)$ for all $v$ has a unique fixed point, $p_{L}$; similarly, considering $f(v)=f_{M}(v)$ and $f(v)=f_{R}(v)$ respectively, we define $p_{M}$ and $p_{R}$. When a limit cycle exists, both $p_{L}$ a $p_{R}$, which have the same Jacobian matrix, lie on the middle zone and so, they are only virtual equilibria; however, their eigenvalues have influence in the dynamics. We denote by $\lambda_{q / s, L / M}$ the eigenvalues of the equilibrium points $p_{L}$ and $p_{M}$ of system (1), where $q$ and $s$ stand for "fast" (eigenvalue with the biggest modulus) and "slow", respectively. To ensure the existence of a periodic orbit, we need to consider the next set of hypotheses:

$$
g_{s y n}>1-\gamma^{-1}, \quad\left|g_{s y n}+C \gamma\right|<1, \quad 0<C \leq C^{*}, \quad \text { and } \quad I_{1}<I<I_{2} \text {, }
$$

where

$$
I_{1}=\left(\frac{a}{2}-v_{s y n}\right) g_{s y n}+\frac{(\gamma+1) a-2 v_{0}+2 \gamma w_{0}}{2 \gamma}, \quad I_{2}=I_{1}+\frac{1}{2} g_{s y n}+\frac{1-\gamma}{2 \gamma} .
$$

With these definitions, we can state the following result.

Theorem 1 Given system (1) under hypothesis (H), for a sufficiently small $C>0$, the period of the unique periodic orbit of the system can be written as $T=\hat{T}+O(C)$, with

$$
\hat{T}=\sum_{j=1}^{2} \frac{1}{\lambda_{s, L}} \ln \left(\left|\frac{\gamma\left(I-I_{j}\right) B_{l}}{\gamma\left(I-I_{j}\right) B_{l}+(-1)^{j} K_{l}}\right|\right)+\frac{1}{\lambda_{q, M}} \ln \left(\left|\frac{\gamma\left(I-I_{j}\right) B_{m}+K_{m}}{\gamma\left(I-I_{j}\right) B_{m}+K_{m, j}}\right|\right),
$$

where 


$$
\begin{aligned}
& B_{m}=\left(\gamma+\lambda_{q, M}\right)\left(\left(G_{s y n}+1\right)\left(\lambda_{s, L}-\lambda_{s, M}\right)-\gamma\left(\lambda_{s, L}+\lambda_{s, M}\right)-2 \lambda_{s, L} \lambda_{s, M}\right), \\
& B_{l}=\lambda_{q, L}-\lambda_{s, L}, \\
& K_{l}=\frac{1}{2}\left(\gamma+\lambda_{q, L}\right)\left(G_{s y n}+\gamma+2 \lambda_{s, L}+1\right), \\
& K_{m}=\frac{1}{2}\left(\gamma+\lambda_{s, M}\right)\left(G_{s y n}-\gamma+1\right)\left(\left(G_{s y n}+1\right)\left(\lambda_{s, L}-\lambda_{q, M}\right)-\gamma\left(\lambda_{s, L}+\lambda_{q, M}\right)-2 \lambda_{s, L} \lambda_{q, M}\right), \\
& K_{m, 1}=\frac{1}{2}\left(\gamma+\lambda_{q, M}\right)\left(G_{s y n}-\gamma+1\right)\left(\left(G_{s y n}+1\right)\left(\lambda_{s, L}-\lambda_{s, M}\right)-\gamma\left(\lambda_{s, L}+\lambda_{s, M}\right)-2 \lambda_{s, L} \lambda_{s, M}\right), \\
& K_{m, 2}=\frac{1}{2}\left(\lambda_{q, M}-\lambda_{s, M}\right)\left(\gamma+\lambda_{q, M}\right)\left(G_{s y n}-\gamma+1\right)\left(G_{s y n}+\gamma+2 \lambda_{s, L}+1\right), \\
& G_{s y n}=g_{s y n} \gamma .
\end{aligned}
$$

Sketch of the proof The period is obtained through straightforward computations which are based on the local linearity of the vector field and the existence of two points, on the switching line, belonging to the periodic orbit. These two points are approximated as the intersection of the slow manifold with the switching line, since the periodic orbit is assumed to pass exponentially close of the slow manifold.

\section{Application to the Estimation of Conductances}

All our numerical tests show evidences that $\hat{T}$ is monotonous with respect to $g_{\text {syn }}$ in the spiking region $I \in\left(I_{1}, I_{2}\right)$; see (2). We have not been able to prove this property yet, but it works for practical purposes and allows us to compute good estimations of slowly-varying synaptic conductances in spiking regimes. It is quite obvious that forcing the system (1) with a steady conductance, the system will exhibit a periodic behaviour of period $T$ and, therefore, solving the equation $\hat{T}\left(C, I, g_{s y n}\right)=T$, we will recover the input synaptic conductance with high accuracy. The challenge, however, is to know whether the conductances can be well estimated when they fluctuate. To test it, we have forced the system (1) with prescribed conductance traces, $g_{s y n}(t)$, and then proceed as follows:

(i) We extract the interspike intervals (distances from maxima) from the resulting voltage trace, thus obtaining a sequence of fluctuating periods $\left\{T_{i}\right\}_{i=1}^{N}$, where $N+1$ is the number of spikes in the simulation.

(ii) For each $i=1, \ldots, N$, we have solved (numerically) the equation $\hat{T}\left(C, I, g_{s y n}\right)$ $=T_{i}$ and choose the positive solution $g_{s y n, i}$ satisfying $I_{1}\left(g_{s y n, i}\right)<I<I_{2}\left(g_{s y n, i}\right)$ to ensure that corresponds to a periodic orbit.

In Fig. 1, we show estimations obtained from three different prescribed inputs: a sinusoidal drive, an oscillatory drive with two frequencies, and a stereotypical synaptic input to a single cell in visual cortex (units scaled to match (1)). We observe fairly good estimations of synaptic conductances (see panels (a), (b), (d) and (e) in Fig. 1). Comparing panels (a) and (b), we observe that, including a higher frequency in the prescribed input, the estimations impoverish. In general, frequencies higher than the sampling frequency are difficult to estimate. However, the reconstructed voltage 
(a)

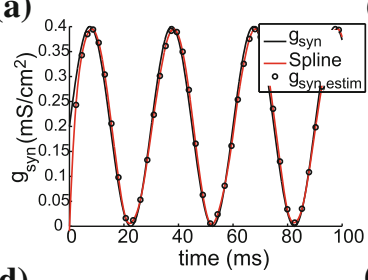

(d)

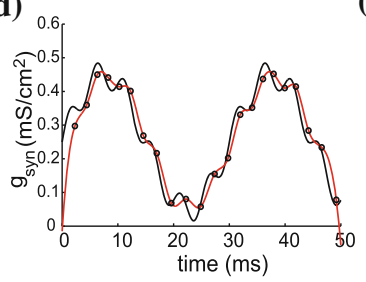

(b)

(e)
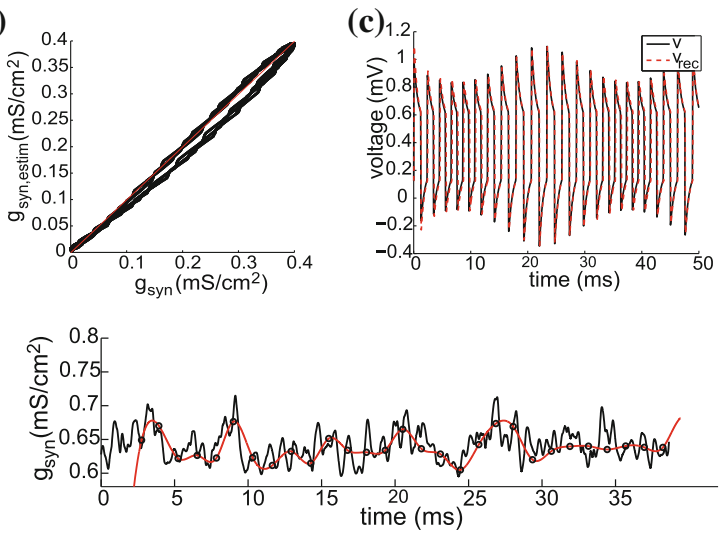

Fig. 1 Estimation of synaptic conductances for different inputs. (a, d, e) estimations of the time course of $g_{s y n}$ when the neuron's synaptic input is a sinusoidal drive, a two-frequency oscillatory drive and a conductance trace taken from a realistic model, respectively; the time courses of prescribed conductances appear in black, open circles indicate the estimates obtained with our algorithm and the spline interpolations of these estimates are plotted in red; (b) estimated values of $g_{s y n}$ against the prescribed values for the sinusoidal drive; (c) voltage trace using the prescribed conductances for the sinusoidal drive (black) compared to the voltage trace reconstructed using the estimated conductances (red)

using the estimated conductance (see panel (c)) exhibits a good agreement in both cases (only sinusoidal drive is shown). Notice that when the prescribed conductances are taken from a realistic model of V1 (see panel (e)), the estimation captures the overall conductance profile but does not match at a smaller scale. In realistic inputs, the conductances' time-scale is variable, being very short in some moments. Thus, we can only get a good estimation on average. A further improvement of our estimation method will consist of introducing more Poincaré sections to estimate flight times in shorter intervals (i.e., refining the sampling).

Acknowledgements This work is partially supported by the Spanish Ministry of Economy and Competitiveness through project MTM2015-71509-C2-2-R (AG), by the MCYT/FEDER grant number MTM2014-54275-P (RP, AT and CV) and by the Government of Catalonia under grant 2014-SGR-504 (AG).

\section{References}

1. L.F. Abbott, A network of oscillators. J. Phys. A: Math General 23(16), 3835 (1990)

2. S. Coombes, Neuronal networks with gap junctions: a study of piecewise linear planar neuron models. SIAM J. Appl. Dyn. Syst. 7(3), 1101-1129 (2008)

3. S. Coombes, R. Thul, K.C.A. Wedgwood, Nonsmooth dynamics in spiking neuron models. Phys. D 241(22), 2042-2057 (2012) 
4. G.B. Ermentrout, D.H. Terman, Mathematical Foundations of Neuroscience (Springer, New York, 2010)

5. S. Fernández-García, M. Desroches, M. Krupa, F. Clément, A multiple time scale coupling of piecewise linear oscillators. Application to a neuroendocrine system. SIAM J. Appl. Dyn. Syst. 14, 643-673 (2015)

6. A. Guillamon, D.W. McLaughlin, J. Rinzel, Estimation of synaptic conductances. J. Physiol.Paris 100(1-3), 31-42 (2006)

7. A. Tonnelier, W. Gerstner, Piecewise linear differential equations and integrate-and-fire neurons: insights from two-dimensional membrane models. Phys. Rev. E 67, 021908 (2003)

8. C. Vich, A. Guillamon, Dissecting estimation of conductances in subthreshold regimes. J. Comput. Neurosci. 39(3), 271-287 (2015) 